\section{Cureus}

Received 07/23/2016

Review began $07 / 28 / 2016$

Review ended 07/30/2016

Published 08/10/2016

\section{(c) Copyright 2016}

Kandi et al. This is an open access article distributed under the terms of the Creative Commons Attribution License CC-BY 3.0., which permits unrestricted use, distribution, and reproduction in any medium, provided the original author and source are credited.

\title{
Emerging Bacterial Infection: Identification and Clinical Significance of Kocuria Species
}

Venkataramana Kandi ${ }^{1}$, Padmavali Palange ${ }^{2}$, Ritu Vaish ${ }^{1}$, Adnan Bashir Bhatti ${ }^{3}$, Vinod Kale

${ }^{4}$, Maheshwar Reddy Kandi ${ }^{5}$, Mohan Rao Bhoomagiri ${ }^{6}$

1. Department of Microbiology, Prathima Institute of Medical Sciences, Karimnagar, IND 2. MD Microbiology, Associate Professor, Department of Microbiology, Prathima Institute of Medical Sciences, Karimnagar, Telangana, IND 3. Medical Director of Clinical Research, Spine Surgery, Tristate Brain and Spine Institute, United States 4. Department of Microbiology, Prathima Institute of Medical Sciences 5. Microbiology, Chalmeda Anandrao Institute of Medical Sciences 6. Department of Microbiology, Prathima Institute of Medical Sciences, Nagunur, IND

$\square$ Corresponding author: Venkataramana Kandi, ramana20021@gmail.com Disclosures can be found in Additional Information at the end of the article

\section{Abstract}

Recently there have been reports of gram-positive cocci which are morphologically similar to both Staphylococci and the Micrococci. These bacteria have been identified as Kocuria species with the help of automated identification system and other molecular methods including $16 \mathrm{~S}$ rRNA (ribosomal ribonucleic acid) evaluation. Kocuria belongs to the family Micrococcaceae which also includes Staphylococcus species and Micrococcus species. Isolation and clinical significance of these bacteria from human specimens warrant great caution as it does not necessarily confirm infection due to their ubiquitous presence, and as a normal flora of skin and mucous membranes in human and animals. Most clinical microbiology laboratories ignore such bacteria as laboratory and specimen contaminants. With increasing reports of infections associated with these bacteria, it is now important for clinical microbiologists to identify and enumerate the virulence and antibiotic susceptibility patterns of such bacteria and assist clinicians in improving the patient care and management. We review the occurrence and clinical significance of Kocuria species.

Categories: Infectious Disease, Pathology

Keywords: kocuria spp, urinary tract infection, micrococcaceae, staphylococcus

\section{Introduction And Background}

Actinobacteria are a unique and large group of bacterial species containing five classes, nineteen orders, 50 families and around 220 genera as revealed by the 16S rRNA studies. They have a rigid cell wall and appear Gram-positive or Gram-variable in Gram's staining. These bacteria are aerobic/facultatively anaerobic and grow better at neutral $\mathrm{pH}$. Many species of Actinobacteria are saprophytic and are present in the environment growing under varied conditions as acidophiles, alkaliphiles, halophiles, and thermophiles. Several other actinobacterial species infect plants and animals. Morphologically Actinobacteria show different shapes ranging from cocci, coccobacilli, bacilli, and long filamentous bacteria.

Kocuria is a Gram-positive cocci arranged in pairs, short chains, tetrads, cubical packets of eight and irregular clusters. Kocuria belongs to the phylum Actinobacteria, class Actinobacteria, order Actinomycetales, sub order Micrococcinae and family Micrococcaceae. This bacterium was first identified and described by Miroslav Kosur, a Slovakian microbiologist. Currently, there are more than 18 species of Kocuria identified based on the 16S rRNA phylogenetic studies. The 


\section{Cureus}

species of Kocuria identified thus far include Kocuria assamensis, Kocuria aegyptia, Kocuria gwangalliensis, Kocuria atrinae, Kocuria carniphila, Kocuria flava, Kocuria palustris, Kocuria halotolerans, Kocuria himachalensis, Kocuria koreensis, Kocuria kristinae, Kocuria marina, Kocuria polaris, Kocuria rhizophila, Kocuria rosea, Kocuria salsicia, Kocuria sediminis, Kocuria turfanensis, and Kocuria varians. Kocuria species (Kocuria spp) inhabit the normal skin and mucous membrane of human and animals [1]. Kocuria was also isolated from various environmental and ecological niches [2]. These are usually considered as non-pathogenic bacteria which are rarely associated with human infections. Recently there has been a rise in the incidence of infections caused by Kocuria spp causing both superficial infections and deep-seated/invasive infections. The cause of concern is that this bacterium appears to have a broad host range involving both immunocompromised as well as immunocompetent individuals. This review attempts to update the morphology, cultural characteristics, pathophysiological properties, and laboratory diagnosis of Kocuria spp.

\section{Review}

\section{Cultural characteristics of Kocuria}

Kocuria spp do not produce hemolysis on blood agar, unlike most clinical isolates of Staphylococci. They usually form 2-3 mm whitish, small, round, raised, convex colonies on initial isolation and might develop non-diffusible yellowish pigmentation after prolonged incubation, as shown in Figure 1.

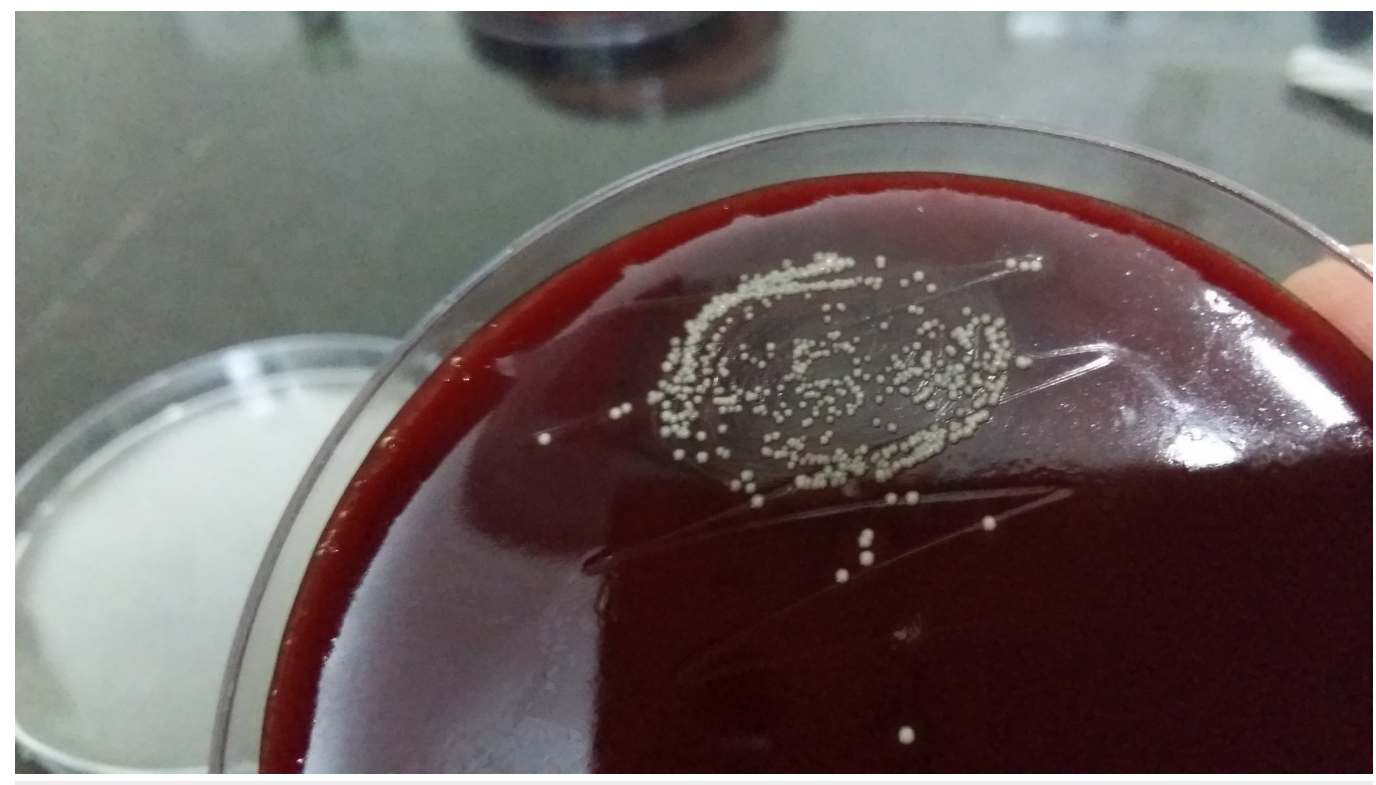

FIGURE 1: Appearance of Kocuria spp on blood agar after 24 hours of aerobic incubation

These bacteria appear large and show both tetrads (Micrococci) and irregular clusters. An interesting observation in Gram's stained smear includes the presence of darkly stained and abnormally large clones of cocci, which are not observed in the case of Staphylococci and Micrococci as shown in Figure 2. 


\section{Cureus}

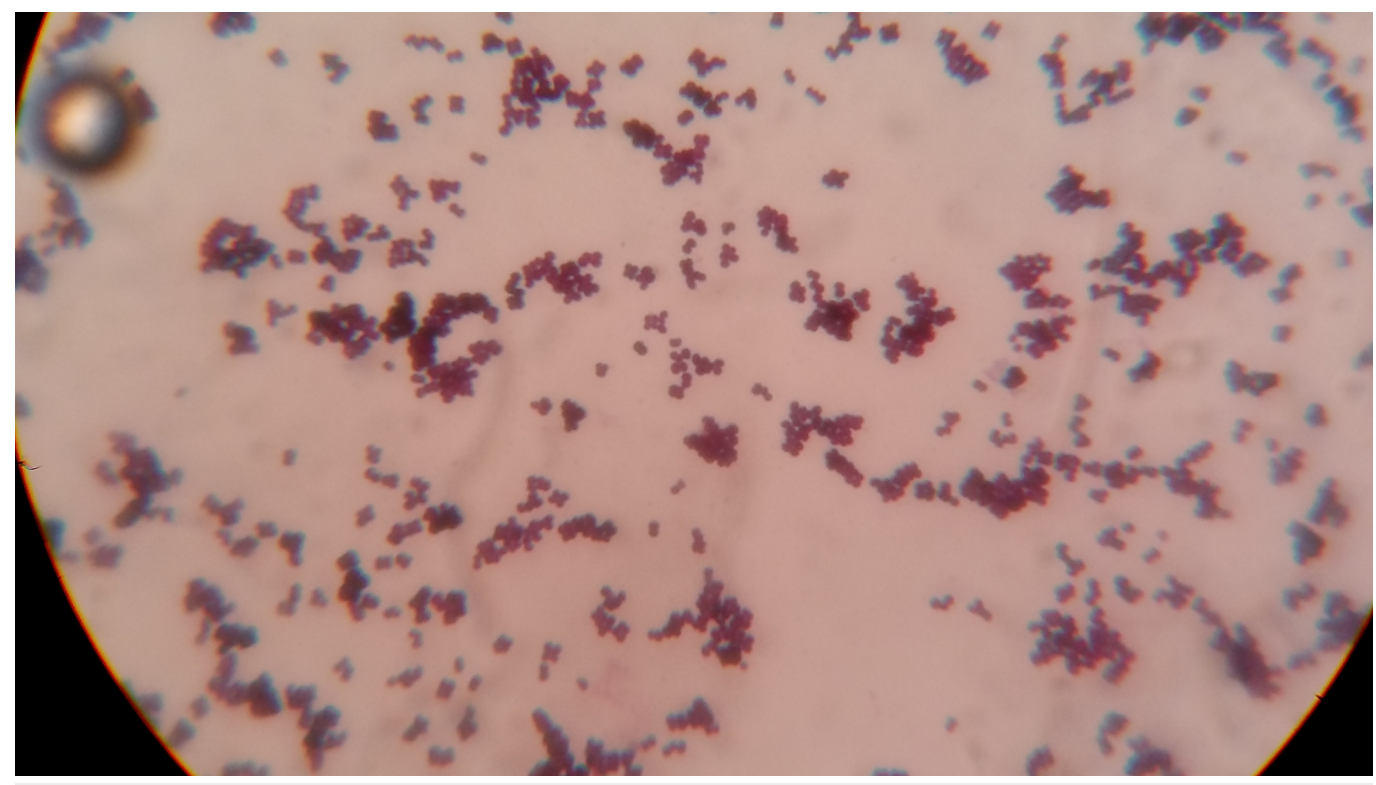

FIGURE 2: Gram's stain of Kocuria spp showing large sized cocci arranged in pairs, short chains, tetrads, clusters and deeply stained very large cocci

Biochemically these bacteria show great variability by reacting differently towards conventional laboratory identification tests including the catalase, urease, and citrate utilization test. These bacteria are normally negative for mannitol fermentation and coagulase enzyme (both bound and free coagulase).

Susceptibility towards bacitracin and lysozyme and resistance to nitrofurantoin, furazolidone and lysostaphin can be used to separate this bacterium from Staphylococci. Modified oxidase test results differentiate between Kicuria spp (negative) and Micrococci.

\section{Clinical profile of Kocuria spp}

Kocuria spp have been reported to be normal flora of human skin and oral cavity and are usually regarded as laboratory contaminants and ignored when isolated in the clinical specimens undermining its pathogenic potential. Kocuria was first identified as a causative agent of urinary tract infection way back in 1974, and it was named as Micrococcus kristinae [3]. Reports of infection with Kocuria species have gained prominence in the late twentieth century and are showing an increased trend, signifying its pathogenic potential. Infections associated with isolation of Kocuria include urinary tract infections, cholecystitis, catheter-associated bacteremia, dacryocystitis, canaliculitis, keratitis, native valve endocarditis, peritonitis, descending necrotizing mediastinitis, brain abscess and meningitis [4-15]. The predisposing factors associated with infections related to Kocuria spp include congenital deformities (short bowel syndrome), chronic catheterization (in cases of total parenteral nutrition), malignancies (ovarian cancer, gastric cancer, myelodysplastic syndrome, acute myelogenous leukaemia, nonHodgkin's disease) and patients with end-stage renal disease undergoing continuous ambulatory peritoneal dialysis. Other underlying conditions associated with Kocuria infection include diabetes mellitus, tuberculosis, stem cell transplant patients, patients suffering from gallstones, methylmalonic aciduria and pancreatic pseudocyst [16-21].

\section{Laboratory identification of Kocuria spp}


This bacterium is normally misidentified in the clinical microbiology laboratories as coagulasenegative Staphylococci (CoNS) based on its gram reaction, catalase positive and coagulase negative properties. Other physiological and biochemical properties of Kocuria are the formations of non-hemolytic colonies on blood agar, non-capsulated, non-spore forming, non-motile, non-acid fast and positive for Voges-Proskauer test (VP). It has also been observed that various species of Kocuria react differently to routine biochemical tests like the oxidase, amylase, urease, citrate utilization test, gelatinase, phosphatase tests, utilization of inulin, arabinose, $\mathrm{N}$-acetyl-L-glutamic acid, and nitrate reduction tests [22]. This could be attributed to the reason behind the inaccurate identification by both conventional and an automated bacterial identification systems.

The major drawback faced by many laboratories in accurately identifying this bacterium is the need for advanced techniques like 16S rRNA and Matrix-Assisted Laser Desorption/Ionization Time-of-Flight Mass Spectrometry (MALDI-TOF-MS). Although many clinical microbiology laboratories are now equipped with automated identification systems that include VITEK (BioMe'rieux Inc., Durham, NC, USA), VITEK 2 (BioMe'rieux Inc., Durham, NC, USA), API (BioMe'rieux Inc., Durham, NC, USA) and the BD Phoenix ${ }^{\mathrm{TM}}$ Automated Microbiology System (BD Diagnostic Systems, Sparks, MD) identification systems, there are studies that have noted false identification of CoNS as Kocuria spp and its limitations to identify all the species of Kocuria [23]. Previous research has highlighted that in the case of non-availability of molecular and advanced laboratory methods, Kocuria can still be identified and differentiated from Staphylococci and Micrococci using morphological, cultural characteristics and differential antibiotic discs. Kocuria spp are sensitive to bacitracin, lysozyme and resistant to nitrofurantoin, furazolidone and lysostaphin [18, 24].

\section{Antimicrobial susceptibility profile of Kocuria spp}

Currently, there are only a few studies that have evaluated the susceptibility profile of Kocuria spp. Antimicrobial susceptibility testing results of random case reports are available, although they are still insufficient to establish the exact susceptibility results of Kocuria [25]. Kocuria spp isolated from a case of peritonitis in a 57 year-old patient suffering from end-stage renal disease revealed sensitivity to ampicillin, cloxacillin, cefotaxime, ciprofloxacin, ofloxacin, levofloxacin, gentamicin, erythromycin, clindamycin, tetracycline, amikacin, linezolid, teicoplanin, vancomycin, imipenem, quinupristin, dalfopristin, rifampicin and was found to be moderately sensitive to ceftazidime [11]. Becker et al. have reported that the Kocuria rhizophila isolated in blood from a case of sepsis in pediatric age patient revealed resistance only to norfloxacin [21]. Studies by Lee et al., who reported multiple cases involving both extreme age groups noted that Kocuria marina isolated from peritoneal fluid was resistant only to tetracycline. Other observations by the same authors showed that $K$. kristinae isolated from bacteremia cases revealed resistance to oxacillin, cefazolin and intermediately sensitive to cefotaxime [11]. Resistance to ciprofloxacin and erythromycin was observed in K. rhizophila isolated from blood of a 3-year-old catheterized patient as reported by Moissenet et al [26]. $K$ varians isolated in peritoneal fluid resistant only to levofloxacin was reported by Meletis et al in a patient undergoing continuous ambulatory peritoneal dialysis (CAPD) [27].

\section{Recent advances}

A recent research report has highlighted the significance of Kocuria in causing hospitalacquired infections [13]. The same study has also noted that although Kocuria spp are commensals of humans, animals and are present in the environment, they should be considered as potential pathogens in patients who are immunocompromised, undergoing critical care treatment and neonates. A study which included 12 pediatric age patients suffering from underlying debilitating conditions like premature birth and cancer had noted that more than $50 \%$ of patients suffered from invasive infections with Kocuria spp [28]. Reports of infections 
caused by Kocuria spp among previously healthy and immunocompetent individuals are showing an increased trend. Kocuria rosea was isolated from a case of descending necrotizing mediastinitis in a 58-year-old woman who was taking medications for gout and hypertension [13]. Another very recent report has observed endocarditis caused by Kocuria rosea in a 10year-old female patient. Although the patient was healthy before suffering from the infection, a history of surgery to correct congenital heart disease was present [9]. Evaluation of biofilm production by Kocuria spp isolated from a case of peritonitis showed that the strain was negative for biofilm production [18]. Isolation of $K$. marina showing tolerance to severe alkaline conditions in a 7-year-old patient receiving epoprostenol therapy should be considered as an alarming signal regarding the potential of Kocuria spp in causing both opportunistic and nosocomial infections [29-30].

\section{Conclusions}

Identification of Kocuria spp remains elusive because most clinical microbiology laboratories have limited or no access to advanced molecular techniques. Laboratory identification of Kocuria spp can be made conventionally only after high laboratory suspicion. Properties such as morphological variability between these bacteria and other similar gram-positive cocci, as well as biochemical properties including the antimicrobial susceptibility patterns against selective antibiotics could be used to presumptively identify Kocuria spp. Infections of Kocuria spp normally involve patients with various debilitated conditions. In the era of drug resistance, and prevalence of multi-drug resistant bacteria, occurrence of Kocuria spp in hospitalized patients should not always be ignored as contaminants. Further studies emphasizing the determination of the virulence, pathogenic potential, predisposing factors and antimicrobial susceptibility patterns of Kocuria spp are warranted.

\section{Additional Information}

\section{Disclosures}

Conflicts of interest: In compliance with the ICMJE uniform disclosure form, all authors declare the following: Payment/services info: All authors have declared that no financial support was received from any organization for the submitted work. Financial relationships: All authors have declared that they have no financial relationships at present or within the previous three years with any organizations that might have an interest in the submitted work. Other relationships: All authors have declared that there are no other relationships or activities that could appear to have influenced the submitted work.

\section{References}

1. Stackebrandt E, Koch C, Gvozdiak O, et al.: Taxonomic dissection of the genus Micrococcus: Kocuria gen. Cohn. nov, 45:682-92. 10.1099/00207713-45-4-682

2. Park EJ, Kim MS, Roh SW, et al.: Kocuria atrinae sp. nov., isolated from traditional Korean fermented seafood. Int J Syst Evol Microbiol. 2010, 60:914-918. 10.1099/ijs.0.014506-0

3. Tvrzová L, Schumann P, Sedlácek I, et al.: Reclassification of strain CCM 132, previously classified as Kocuria varians, as Kocuria carniphila sp. nov. Int J Syst Evol Microbiol. 2005, 55:139-142. 10.1099/ijs.0.63304-0

4. Mashouf RY, Babalhavaeji H, Yousef J: Urinary tract infections: bacteriology and antibiotic resistance patterns. Indian Pediatr. 2009, 46:617-620.

5. Ma ES, Wong CL, Lai KT, et al.: Kocuria kristinae infection associated with acute cholecystitis . BMC Infec Dis. 2005, 5:60. 10.1186/1471-2334-5-60

6. Sohn KM, Baek JY, Kim SH, et al.: Catheter-related bacteremia caused by Kocuria salsicia: the first case. J Infect Chemother. 2015, 21:305-7. 10.1016/j.jiac.2014.11.005

7. Domont F, Fleche-Mateos AL, Bremond-Gignac D, et al.: Kocuria dacryocystitis infection, caused by Kocuria ocularis sp. Nov. JMM Case Rep. 2014, 1:1-4. 10.1099/jmmcr.0.002022

8. Mattern RM, Ding J: Keratitis with Kocuria palustris and Rothia mucilaginosa in Vitamin A 
Deficiency. Case Rep Ophthalmol. 2014, 5:72-77. 10.1159/000360391

9. Moreira JS, Riccetto AG, Silva MT, et al.: Endocarditis by Kocuria rosea in an immunocompetent child. Braz J Infect Dis. 2015, 19:82-4. 10.1016/j.bjid.2014.09.007

10. Srinivasa KH, Agrawal N, Agarwal A, et al.: Dancing vegetations: Kocuria rosea endocarditis . BMJ Case Rep. 2013, 2013:2013010339. 10.1136/bcr-2013-010339

11. Lee JY, Kim SH, Jeong HS, et al: Two cases of peritonitis caused by Kocuria marina in patients undergoing continuous ambulatory peritoneal dialysis. J Clin Microbiol. 2009, 47:3376-3378. 10.1128/JCM.00847-09

12. Brändle G, L'Huillier AG, Wagner N, et al.: First report of Kocuria marina spontaneous peritonitis in a child. BMC Infect Dis. 2014, 14:719. 10.1186/s12879-014-0719-5

13. Lee MK, Choi SH, Ryu DW: Descending necrotizing Mediastinitis caused by Kocuria rosea: a case report. BMC Infectious Diseases. 2013, 13:475. 10.1186/1471-2334-13-475

14. Tsai CY, Su SH, Cheng YH, et al.: Kocuria varians infection associated with brain abscess: A case report. BMC Infect Dis. 2010, 10:102. 10.1186/1471-2334-10-102

15. Sipahi OR, Mermer S, Aydemir S, et al.: Kocuria rosea meningitis. Surg Infect (Larchmt. 2014, 15:659. 10.1089/sur.2013.220

16. Martinaud C, Gaillard T, Brisou P, et al.: Bacteremia caused by Kocuria kristinae in a patient with acute leukaemia. Med Maladies Infect. 2008, 38:165-6. 10.1016/j.medmal.2007.11.006

17. Lee M-N, Huh HJ, Kim B, et al.: A case of catheter-related Kocuria marina bloodstream infection in a patient with multiple myeloma. Lab Med Online. 2014, 2014:51-5.

10.3343/lmo.2014.4.1.51

18. Purty S, Saranathan R, Prashanth K, et al: The expanding spectrum of human infections caused by Kocuria species: a case report and literature review. Emerg Microbes Infect. 2013, 2:71. 10.1038/emi.2013.71

19. Citro R, Prota C, Greco L, et al.: Kocuria kristinae endocarditis related to diabetic foot infection. J Med Microbiol. 2013, 62:932-4. 10.1099/jmm.0.054536-0

20. Altuntas F, Yildiz O, Eser B, et al.: Catheter-related bacteremia due to Kocuria rosea in a patient undergoing peripheral blood stem cell transplantation. BMC Infect Dis. 2004, 4:62. 10.1186/1471-2334-4-62

21. Becker K, Rutsch F, Uekötter A, et al.: Kocuria rhizophila adds to the emerging spectrum of micrococcal species involved in human infections. J Clin Microbiol. 2008, 46:3537-9. 10.1128/JCM.00823-08

22. Savini V, Catavitello C, Masciarelli G, et al.: Drug sensitivity and clinical impact of members of the genus Kocuria. J Med Microbiol. 2010, 59:1395-402. 10.1099/jmm.0.021709-0

23. Boudewijns M, Vandeven J, Verhaegen J, et al.: Vitek 2 automated identification system and Kocuria kristinae. J Clin Microbiol. 2005, 43:5832. 10.1128/JCM.43.11.5832.2005

24. Ben-Ami R, Navon-Venezia S, Schwartz D, et al.: Infection of a ventriculoatrial shunt with phenotypically variable Staphylococcus epidermidis masquerading as polymicrobial bacteremia due to various coagulase-negative Staphylococci and Kocuria varians. J Clin Microbiol. 2003, 41:2444-2447. 10.1128/JCM.41.6.2444-2447.2003

25. Szczerba I: [Susceptibility to antibiotics of bacteria from genera Micrococcus, Kocuria, Nesterenkonia, Kytococcus and Dermacoccus]. Med Dosw Mikrobiol. 2003, 55:75-80.

26. Moissenet D, Becker K, Mérens A, et al.: Persistent bloodstream infection with Kocuria rhizophila related to a damaged central catheter. J Clin Microbiol. 2012, 50:1495-8. 10.1128/JCM.06038-11

27. Meletis G, Gogou V, Palamouti M, et al.: Catheter-related relapsing peritonitis due to Kocuria varians in a patient undergoing continuous ambulatory peritoneal dialysis. Nefrología (Madr. 2012, 32:541-2. 10.3265/Nefrologia.pre2012.Apr.11471

28. Chen HM, Chi H, Chiu NC, et al.: Kocuria kristinae: a true pathogen in pediatric patients . J Microbiol immunol infect. 2015, 48:80-84. 10.1016/j.jmii.2013.07.001

29. Horiuchi A, Kubota N, Hidaka E, et al.: Notable alkaline tolerance of Kocuria marina isolate from blood of a pediatric patient with continuous intravenous epoprostenol therapy. J Infect Chemother. 2015, 21:680-686. 10.1016/j.jiac.2015.06.004

30. Dotis J, Printza N, Papachristou F: Peritonitis attributable to Kocuria rosea in a pediatric peritoneal dialysis patient. Perit Dial Int. 2012, 32:577-8. 10.3747/pdi.2011.00300 